\title{
ANISOTROPIE PARAMAGNÉTIQUE EN CHAMP FORT ET ANISOTROPIE DE L'ÉCHANGE ; APPLICATION AU DYSPROSIUM
}

\author{
P. BOUTRON, J. L. FERON, G. HUG et P. MORIN \\ Laboratoire d'Electrostatique et de Physique du Métal C. N. R. S., \\ Cedex 166, Grenoble, France
}

Résumé. - Nous avons mesuré la susceptibilité paramagnétique du dysprosium en fonction du champ jusqu'à $85 \mathrm{kOe}$; elle varie comme $H^{2}$ le carré du champ. Nous avons constaté que ce terme en $H^{2}$ est anisotrope. Il a deux origines : la magnétostriction et le terme d'ordre un en $1 / T$ du développement de l'inverse de la susceptibilité au-dessus du point d'ordre. Cette dernière contribution, si on sait évaluer la magnétostriction, permet d'accéder à l'anisotropie de l'échange. Celle-ci ne pourrait pas être déterminée par l'anisotropie de la susceptibilité initiale qui dépend aussi de l'anisotropie à un ion.

Abstract. - The paramagnetic susceptibility of dysprosium has been measured as a function of the magnetic field up to $85 \mathrm{kOe}$; it varies as $H^{2}$, the square of the field. We have observed that this term in $H^{2}$ is anisotropic. It is issued from magnetostriction and from the first order term in $1 / T$ of the expansion of the reciprocal susceptibility above the order point. This last contribution, if we know how to evaluate the magnetostriction, allows us to calculate the exchange anisotropy that could not be determined from the anisotropy of the initial susceptibility also depending of one ion anisotropy.

La susceptibilité d'une substance dans le domaine paramagnétique contient un terme variant comme le rapport $H^{2} / T$ dont la détermination nécessite des champs magnétiques forts. Le coefficient de ce terme dépend de l'énergie d'échange correspondant à la direction $\mathrm{du}$ champ appliqué et des variations des paramètres du cristal. L'anisotropie de ce coefficient peut conduire à l'évaluation de l'anisotropie de l'échange, en particulier dans les terres rares.

RÉSUltats EXPÉRIMENTAUX. - Nous avons étudié la susceptibilité d'un monocristal de dysprosium suivant l'axe $\mathrm{c}$ et l'axe a entre 0 et $85 \mathrm{kOe}$ à 220,240 , 260 et $280^{\circ} \mathrm{K}$. Ce domaine de température est assez éloigné de la température de Néel du dysprosium, $180^{\circ} \mathrm{K}$. Les variations de la susceptibilité avec le champ sont faibles; une grande précision tant sur l'aimantation que sur la température est nécessaire. Pour mesurer les aimantations, nous avons utilisé un voltmètre intégrateur numérique donnant une précision relative sur l'aimantation comprise entre $2 \times 10^{-3}$ pour les plus faibles aimantations et $2 \times 10^{-4}$ pour les plus fortes. La température de l'échantillon est maintenue à $\pm 0,05 \circ \mathrm{K}$; le champ est déterminé avec une erreur relative inférieure à $2 \times 10^{-4}$.

Nous avons représenté sur la figure la variation expérimentale de la susceptibilité avec le carré du champ à $260^{\circ} \mathrm{K}$; cette variation est de la forme

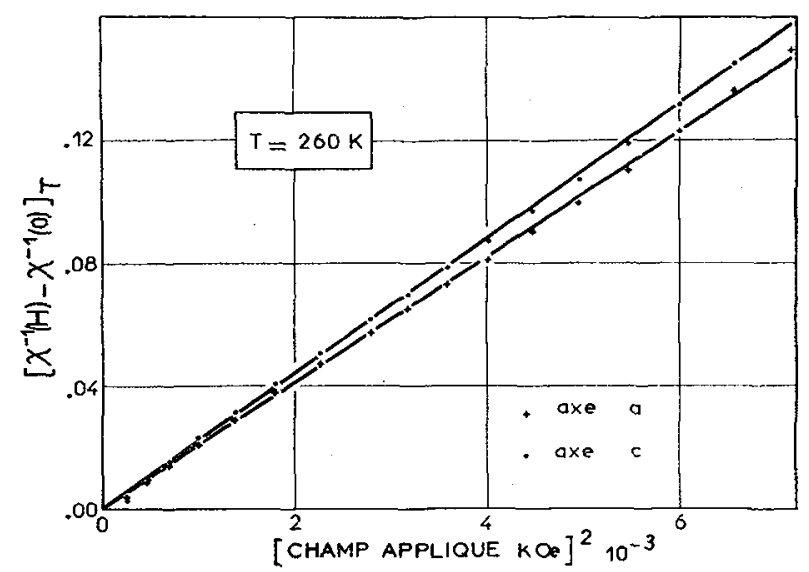

Variation de l'inverse de la susceptibilité en fonction du champ.

$$
\begin{aligned}
& \frac{1}{\chi_{z}}(T, H)-\frac{1}{\chi_{z}}(T, 0)=A_{z} H^{2} \\
& \frac{1}{\chi_{x}}(T, H)-\frac{1}{\chi_{x}}(T, 0)=A_{x} H^{2}
\end{aligned}
$$

lorsque le champ est appliqué respectivement suivant l'axe c est dans le plan de base. Les valeurs de $A_{z}$ et $A_{x}$ sont portées dans le tableau.

EFFET de la magnétostriction, - Dans le domaine paramagnétique, la magnétostriction contribue à la variation de la susceptibilité avec le champ. En effet, la susceptibilité $\chi$, fonction de l'énergie d'échange et $\mathrm{du}$ potentiel cristallin, dépend des paramètres $\mathrm{du}$ cristal qui dépendent eux-mêmes du champ appliqué. L'équilibre du cristal est obtenu en minimisant son énergie libre $\mathfrak{F}$. Dans le domaine paramagnétique, en champ nul, $\mathscr{F}$ est identique à l'énergie élastique. Les paramètres et la température du cristal étant fixes, si le champ varie, $\mathrm{d} \mathscr{F}=-\mathbf{M} \mathbf{d H}$. On obtient l'expression de $\mathscr{F}$ en présence de champ par intégration

$$
\begin{aligned}
\mathscr{F}(a, b, c, \mathbf{H}) & =\frac{1}{2} C_{11}\left(e_{a a}^{2}+e_{b b}^{2}\right)+\frac{1}{2} C_{33} e_{c c}^{2} \\
+ & C_{12} e_{a a} e_{b b}+C_{13}\left(e_{a a} e_{c c}+e_{b b} e_{c c}\right) \\
& -\frac{1}{2} \chi(a, b, c) H^{2}
\end{aligned}
$$

$C_{i j}$ sont les coefficients élastiques, $e_{i j}$ les déformations.

Les dérivées de la susceptibilité par rapport aux paramètres $\mathrm{du}$ cristal sont obtenues en fonction des déformations en annulant les dérivées de $\mathcal{F}$ par rapport aux paramètres. Clark a mesuré ces déformations par jauges de contraintes [1]. Dans le domaine paramagnétique, elles varient, en première approximation comme le carré de l'aimantation

$$
\begin{aligned}
& \left\{\begin{array}{l}
e_{a a, x}=-0,92 \times 10^{-12} M_{x}^{2} \\
e_{b b, x}=-2,68 \times 10^{-12} M_{x}^{2} \\
e_{c c, x}=3,34 \times 10^{-12} M_{x}^{2}
\end{array}\right. \\
& \left\{\begin{array}{l}
e_{a a, z}=e_{b b, z}=-3,20 \times 10^{-12} M_{z}^{2} \\
e_{c c, z}=5,57 \times 10^{-12} M_{z}^{2}
\end{array}\right.
\end{aligned}
$$

$M_{x}$ et $M_{z}$ sont en u. e. m./at. g. Cette variation des déformations comme $M_{x}^{2}$ et $M_{z}^{2}$ était prévisible. En effet, elles s'expriment en fonction des dérivées de la 
susceptibilité (6) qui ne dépendent que du carré de la susceptibilité initiale. La contribution de la magnétostriction à la susceptibilité

$$
\chi_{\mathrm{ms}}=\frac{\partial \chi}{\partial e_{a a}} e_{a a}+\frac{\partial \chi}{\partial e_{b b}} e_{b b}+\frac{\partial \chi}{\partial e_{c c}} e_{c c}
$$

est évaluée à l'aide des déformations (4). Il en résulte une contribution $A_{\mathrm{ms}}(T) H^{2}$ à l'inverse de la susceptibilité. Les valeurs de $A_{\text {ms }}$ lorsque le champ est suivant l'axe c ou dans le plan de base, sont portées dans le tableau; elles sont du même ordre de grandeur que les valeurs expérimentales $A$ mais de signe opposé.

EXPRESSION DE LA SUSCEPTIBILITÉ DANS L'HYPOTHÈSE DU CHAMP MOLÉCULAIRE. ANISOTROPIE DE L'ÉCHANGE. - On peut rendre compte simplement de l'anisotropie de l'échange en le considérant comme quadratique. Le champ moléculaire est alors relié tensoriellement à l'aimantation. Le champ effectif agissant sur un atome a alors pour expression

$$
\mathbf{H}_{\text {eff }}=\mathbf{H}+\overline{\bar{n}} \mathbf{M} \text {; }
$$

$\overline{\bar{n}}$ est un tenseur symétrique. Pour connaître la dépendance avec le champ de l'inverse de la susceptibilité, il est nécessaire d'en poursuivre le développement jusqu'à l'ordre un en $1 / T[2,3]$

$$
\begin{aligned}
\frac{1}{\chi_{i}}(T, H)=\frac{1}{C}\left[T-n_{i} C\right. & +\frac{(2 J+1)(2 J+3)}{5 k} u_{2 i}^{0}+ \\
& \left.+\frac{1}{T}\left(a_{i}+A_{\mathrm{ex}, i} H^{2}\right)\right]
\end{aligned}
$$

avec $A_{\text {ex }, i}=b\left[n_{i} \chi_{i}+1\right]^{2}$.

L'indice $i$ désigne $x$ ou $z$; pour les terres rares $u_{2 x}^{0}=-u_{2 z}^{0} / 2 ;$ pour le dysprosium $b=3,4 \times 10^{-8}$.

En tenant compte de la magnétostriction, on peut accéder, par l'intermédiaire des coefficients de champ moléculaire $n_{x}$ et $n_{z}$, à l'échange et à son anisotropie.

Nous avons reporté dans le tableau, à titre indicatif, les valeurs de $n_{x}$ et $n_{z}$ estimées à partir des variations expérimentales de la susceptibilité avec le champ et des valeurs des déformations mesurées par Clark. Ces valeurs correspondent à une anisotropie de l'échange relativement importante; elles sont inférieures à la valeur moyenne $n=\theta / C=10,4$ u. e. m./at.g déduite de la température de Curie paramagnétique moyenne $\theta=1 / 3 \theta_{z}+2 / 3 \theta_{x}$.

Pour mieux préciser l'anisotropie de l'échange des terres rares, il serait nécessaire d'améliorer tant le modèle théorique que la précision de l'évaluation expérimentale du terme magnétostrictif. Par la méthode des jauges de contraintes, les déformations ne sont pas connues avec une précision suffisante. Il serait préférable de mesurer directement les variations de la susceptibilité avec les contraintes. Pour rendre compte de l'anisotropie de l'échange, le modèle de champ moléculaire n'est peut-être pas assez précis d'autant plus que l'application d'un champ peut induire un ordre à courte distance. La validité du champ moléculaire pourrait être précisée par des mesures analogues sur le gadolinium. On peut remarquer, aussi, que l'échange dans les terres rares contient à la fois des termes quadratiques et biquadratiques [4].

\section{TABLEAU}

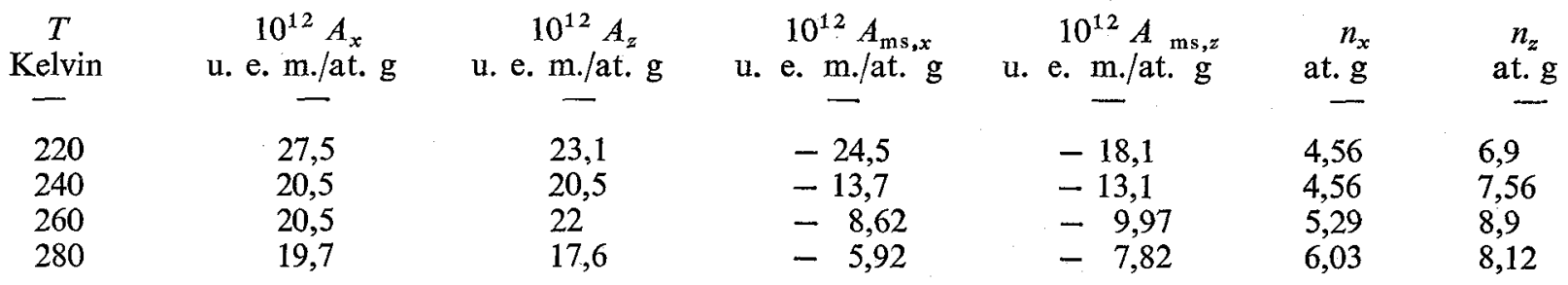

Bibliographie

[1] Clark (A. E.), Desavage (B. F.), Bozorth (R. M.), Phys. Rev., 1965, 138, $1 \mathrm{~A}, 216$.

[2] Aleonard (R.), Boutron (P.), BloCh (D.), J. Phys. Chem. Solids, $1969,30,2277$.

[3] Boutron (P.), J. Physique, 1969, 30, 413.

[4] SPeCht (F.), Phys. Rev., 1967, 162, 389. 